\title{
RADIO GALAXIES AND QUASARS AS COSMOLOGICAL PROBES
}

\author{
ASHOK K. SINGAL \\ Netherlands Foundation for Research in Astronomy \\ Postbus 2, 7990 AA Dwingeloo, The Netherlands \\ e-mail: singal@nfra.nl
}

\begin{abstract}
Various techniques and methods that have been developed for using extragalactic radio sources as cosmological probes of the universe are listed. The discussion is mainly confined to the cosmological tests employing extended radio sizes of powerful radio galaxies and quasars as standard metric rods (in a statistical sense) to figure out the geometry of the universe. Some comments are made on the recent use of the milliarcsec scale sizes of compact radio sources for angular size-redshift tests. It is further pointed out that the estimates of clustering for quasars selected at centimetre wavelengths could be seriously affected by the relativistic beaming.
\end{abstract}

\section{Introduction}

Right in the early years of radio astronomy it became apparent that the population of extragalactic radio sources consists of extremely powerful objects, visible up to large cosmological distances. It gave rise to the early hope that by using these sources as deep probes into the universe we might be able to discriminate between various cosmological world-models. These hopes were somewhat belied when the subsequent studies showed that in addition to the presence of a large statistical spread in the intrinsic luminosities and physical sizes of extragalactic radio sources, even the average properties of their population seem to evolve heavily with the cosmic epoch and that the evolution almost completely masks out any distinguishable features of geometry between various world-models. However, a study of the cosmological evolution of the various properties of these radio sources is in itself of interest as it may yield information on the conditions that prevailed 71

M. Kafatos and Y. Kondo (eds.), Examining the Big Bang and Diffuse Background Radiations, 71-78. (c) 1996 IAU. Printed in the Netherlands. 
in the cosmos at different epochs. In addition, some other techniques have now emerged that make use of the radio sources for cosmological investigations without relying critically upon their above evolving properties.

The following techniques and methods exploit radio galaxies and quasars as direct or indirect probes for the study of various cosmological questions.

\section{Classical Methods}

1. Angular distribution across the sky : looking for departures from an isotropic distribution on large angular scales - a consistency check for the Cosmological Principle.

2. Hubble diagram : The possibility to identify radio galaxies at high redshifts $(z \geq 1)$ and the relatively small scatter seen in their $K-z$ plots could revive a further interest in one of this most classical cosmological test.

3. Radio Source Counts : Yield information on the space distribution of sources and the cosmological evolution of their number densities and/or of their radio luminosities.

4. Radio size distribution across the luminosity-redshift plane : looking for any signature of the curvature of the universe from a variation of the median value of their angular sizes with redshift. Interpretation complicated due to a cosmological evolution of their sizes and/or the presence of a luminositysize correlation.

\section{Other Methods}

5. High redshift radio galaxies and quasars : These provide lower limits on the epoch of galaxy formation and further, yield information on the properties of the intervening matter from the absorption spectra of high redshift quasar.

6. Gravitational lenses: $H_{0}$ from the arrival times in gravitationally-lensed multiple images of a source.

7. Superluminal expansions: $H_{0}$ (and also $q_{0}$ !) from the observed proper motions of the VLBI jet-components.

8. Pressure and energy-density in the lobes of giant radio galaxies and quasars: Possibility of putting some constraints on the physical conditions in the intergalactic medium (IGM).

A brief review of most of these topics with references is available in Shaver (1992), which I will not repeat here. Also, some of these topics are being covered in detail by others in the present proceedings. My discussion will mainly be confined to the observed size distributions of radio galaxies and quasars across the luminosity-redshift plane. But first I would like to 
make a few comments about the amount of clustering inferred for quasars selected at centimetre wavelengths.

Quasars may appear to be the ideal objects for studying very large scale structures in the universe. There have been many such studies based on optically selected quasar samples (see e.g. Komberg et al. 1994 and the references therein). Some of the investigators have used very heterogeneous samples, which contained a very mixed sample of optically as well as radio selected quasars (see e.g. Shaver 1988 and the references therein). The spatial clustering of quasars seems to have been confirmed at high significant levels (see Shaver 1992). There are even reports that the radio-selected quasars appear to have larger correlation amplitude than the other type of quasars (Chu and Zhu 1988). Here I will like to draw attention to an effect which may cause the two-point correlation function inferred for radio quasars, selected generally at centimetre wavelengths, to be an underestimate. If we do believe that the flat spectrum quasars get selected mostly by their "beamed" cores (as first suggested by Orr and Browne 1982), then we do not expect to see many physically associated close pairs (provided they do exist) to show up in samples selected at high frequencies which are dominated by flat spectrum quasars. It is very unlikely that both members of a pair will be beamed towards the same observer and thus even if large number of quasar pairs do exist the probability of finding them this way appears to be very low. It appears more logical that one should look for such pairs in metre-wavelength selected samples where quasars are selected mostly by their extended lobe emission. The only available such complete sample at metre wavelength is the 3CRR (Laing et al. 1983), unfortunately it contains only a small number of quasars. The absence of sufficient number of known steep-spectrum quasars in metre-wavelength selected samples in the first place is the very reason why people went for flat spectrum quasars. Recently Kapahi et al. (1994) have formed a somewhat larger sample of metre-wavelength selected quasars, called the Molonglo Quasar Sample. Unfortunately this sample is confined to a narrow strip in the sky, but one could still try to look for clustering in such samples.

\section{Extended radio sizes as cosmological tools}

The usage of the extended sizes of radio galaxies and quasars for cosmological investigations first began with Miley $(1968,1971)$ and Legg $(1970)$ who showed that there appears to be a deficiency of large sized radio quasars at higher redshifts. In fact the upper envelope of the angular size-redshift $(\theta-z)$ distribution in Miley's (1971) plot seems to fit well with a function $\theta \propto z^{-1}$, which in the literature has often been "loosely" described as what would be expected in a static-Euclidean universe. I may point out here 
that in a static-Euclidean universe, by definition, there is no cosmological expansion. Consequently, the observed redshift of a source has no simple cosmological interpretation and it may bear no direct relation to the "distance" of the source (except perhaps in a "tired-light effect" type model). Therefore, a relation of the type $\theta \propto z^{-1}$ is not something to be normally expected in a static-Euclidean universe.

In an alternative approach, Swarup (1975) and Kapahi (1975) investigated the variation in the median value of the angular size distribution with a decreasing flux density level of the source sample. One of the main advantages of this approach at that time was that the sample was not necessarily limited to a small number of known-redshift sources as the redshift information for the whole sample is not a prerequisite in this technique. However, for a proper interpretation one does need to assume a luminosity function for the radio source population, adding to the uncertainties.

Both kinds of above studies showed the inadequacies of constant physical size models for the population of extragalactic radio sources and the inference generally drawn was that the sources had smaller physical sizes at earlier epochs. However, a suitable luminosity-linear size $(P-l)$ correlation among the radio source population could also explain these observations, without invoking the size evolution. And there is a possibility that both the size evolution and the luminosity-size correlation could be present. The only reliable way to separate these two effects is to investigate the size distribution in the luminosity-redshift plane, where one could not only examine the $l-z$ relation for a given luminosity class, but could also check for a $P-l$ correlation in a given redshift bin. In addition, one also avoids the need to know an appropriate luminosity function.

The main requirement for undertaking such an investigation is to have a large enough sample so that relevant portions of the $P-z$ plane could be populated with statistically significant number of sources in various bins. One needs information on the flux-density, spectral index, angular size and redshift for each source in the sample. Moreover the sample has to be "fare" in the sense that no selection bias based on a prior information on the angular sizes of the souces has crept in. We may point out here that contrary to the view expressed sometimes in the literature it is neither needed nor desired to have a 'radio complete' sample for the above purpose. In fact, paradoxical as it may sound, the condition of the strict radio-completeness of the chosen sample, a must for number counts and other such studies, may somewhat be of a disadvantage for the present purpose. Such a flux-limited radio complete sample covers only a narrow band in the $P-z$ plane. In order to fill the luminosity-redshift plane more evenly, we are forced to employ data from many sub-samples selected at different flux-density limits, which will make our final sample to be far from a radio-complete one. 


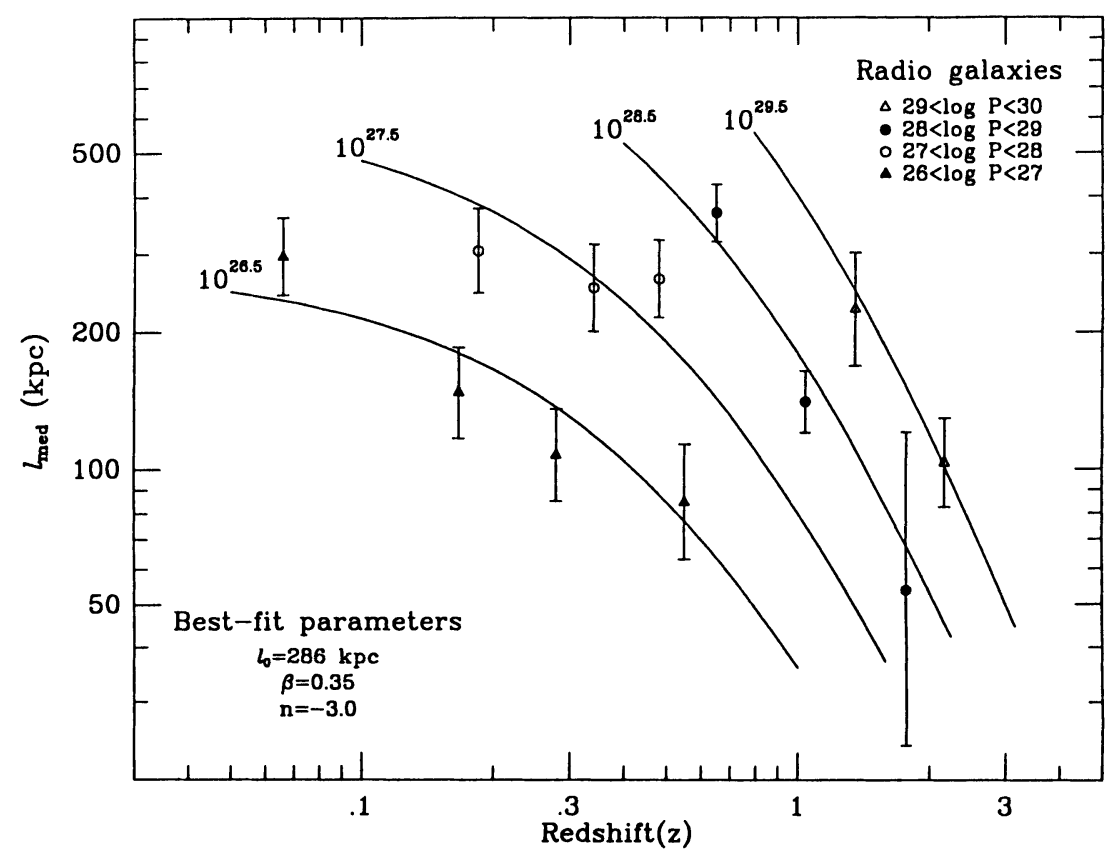

Figure 1. The change in $l_{\text {med }}$ with redshift for radio galaxies. The family of curves is drawn according to the relation $l=l_{0}\left(P / 10^{26.5}\right)^{\beta}(1+z)^{n}$, for the best-fit parameter values $l_{0}=286 \mathrm{kpc}, \beta=0.35, n=-3$ (Singal 1993).

Following such an approach, many independent workers (although their data used were not entirely independent) showed (Oort et al. 1987; Singal 1988; Kapahi 1989) that the physical sizes of the radio galaxies appear to evolve rapidly with redshift $\left(l \propto(1+z)^{-3}\right)$. In addition, Singal (1988) also pointed out that the size evolution of quasars, if any, appears to be much weaker (see also Barthel and Miley 1988). This unexpected difference between radio galaxies and quasars was later confirmed by Singal (1993) from a larger sample of sources that included many more galaxies at high redshifts. Using a multiple regression analysis with the functional form $l \propto P^{\beta}(1+z)^{n}$ on a large sample of 789 powerful $\left(P_{408} \geq 10^{26} \mathrm{~W} \mathrm{~Hz}^{-1}\right.$ for $\left.H_{0}=50 \mathrm{~km} \mathrm{~s}^{-1} \mathrm{Mpc}^{-1}, q_{0}=0\right)$ radio sources, Singal (1993) arrived at the following conclusions (see Figures 1 and 2 here):

1. There is a direct correlation between luminosity and size for radio galaxies $(\beta \simeq 0.35)$ for a given redshift.

2. The radio size of galaxies in a given luminosity range falls sharply $(n \simeq-3.0)$ with the cosmic epoch. 


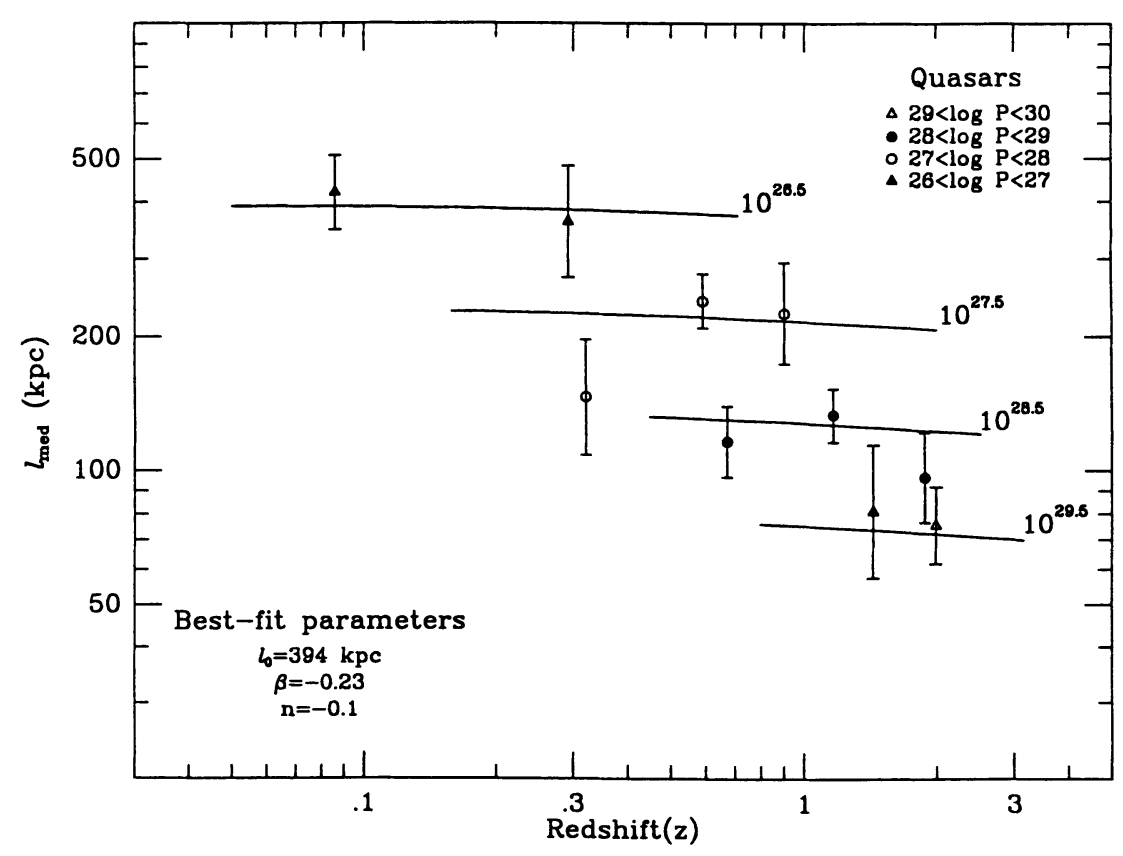

Figure 2. The size evolution for quasars. The family of curves is drawn according to the relation $l=l_{0}\left(P / 10^{26.5}\right)^{\beta}(1+z)^{n}$, for the best-fit parameter values $l_{0}=394 \mathrm{kpc}$, $\beta=-0.23, n=-0.1$ (Singal 1993).

3. There is an inverse correlation between luminosity and size $(\beta \sim-0.2)$ for quasars, in contrast with the case of galaxies.

4. For quasars, the cosmic size evolution, if any, is only marginal $(n \sim-0.2)$.

The large differences seen between radio galaxies and quasars in the redshift and luminosity dependences of their sizes, provide strong evidence against the simple orientation based unified-scheme models (Barthel 1989). Further, the task of explaining the size evolution and/or the luminosity-size correlation by a physical model (see e.g. Gopal-Krishna and Wiita 1991) becomes even more difficult. We may here add that there appeared to be some confusion in the above picture when Nilsson et al. (1993) claimed that in their studies they find no significant difference between the radio sizes of radio galaxies and quasars. They also reported the presence of a negative correlation between radio size and power among high luminosity radio galaxies, a result in contradiction with almost all other previous studies. A comparison of Figure 3 of Nilsson et al. (1993) with Figures 1 and 2 here (as reproduced from Singal 1993) does not show much discordant 
data between the two samples in the regions of overlap but it does show clearly that the conclusions of Nilsson et al. suffer from a lack of data on high redshift galaxies. Further, it is not clear how much bias could have got introduced in the size distribution due to their insistence on retaining only "well-defined" doubles in their final source sample, as such a selection effect is likely to discriminate against small-sized but high redshift sources. Recently Kapahi et al. (1994) have investigated the size distribution in their Molonglo Quasar Sample and their results indicate a clear difference in the luminosity-size correlation between radio galaxies and quasars, in agreement with the conclusions of Singal (1993).

\section{Compact radio sources}

The failure of the extended structures of extragalactic sources to provide good yard-sticks for measuring the geometry of the universe has led to a recent growth of interest in the use of milli-arcsec sizes of the compact radio sources for determining the deceleration parameter $q_{0}$ (Kellermann 1993; Gurvits 1994). The main argument here is that since these compact structures lie well within the host galaxy environment, their sizes should not be directly affected by a change in the density of the IGM with the cosmological expansion and as a result are less likely to be evolving with redshift. Using a sample of 82 compact radio sources, taken from the literature, Kellermann (1993) found that his angular size-redshift plot supports the Einstein-de Sitter universe with $q_{0}=0.5$. On the other hand Gurvits (1994) finds the data on milli-arcsec sizes to be somewhat more consistent with an open universe.

Unfortunately, the usage of milli-arcsec structures of compact sources brings its own bigger bag of problems. The first and the foremost difficulty is that unlike in the case of extended structures, where hot-spots could be seen, the milli-arcsec scale VLBI jets do not possess well recognizable extremities and it becomes very difficult to give an unambiguous size estimate which is independent of the observing technique (frequency of observations, the achieved sensitivity etc.). It is not even clear whether the measured size reflects the same intrinsic property that is stable from source to source (Pearson et al. 1994). In this context it is not clear whether a sizable fraction of sources which may remain unresolved should be included as upper limits on sizes. The altogether absence of a VLBI scale jet is not the same thing as the jet being smaller in size than the observing beam. We can not even be very sure that evolution plays no significant role in VLBI scale jets (even if these may not be directly influenced by the IGM). Then there is a strong likelihood of the relativistic beaming introducing large selection effects. Any small changes in the orientation with respect to 
the observer may introduce large changes in the estimates of the projected sizes. In the angular size-redshift plot of Pearson et al. (1994) there is no evidence of any dependence of angular size with redshift. In fact their data disagree with those of Kellermann (1993) in the lowest-redshift bin which unfortunately happens to provide the most crucial point in Kellermann's estimate of $q_{0}$. To be fare, it is not clear how much this disagreement may be arising from the difference in the luminosity levels of the two samples. It is to be hoped that in the next few years the large number of VLBI maps expected to come out from the VLBA and EVN (European VLBI Network) may help resolve some of these important issues.

\section{References}

Barthel, P. D., 1989, ApJ, 336, 606

Barthel, P. D., Miley, G. K., 1988, Nature, 333, 319

Chu, Y., Zhu X, 1988, A\&A, 205, 1

Gopal-Krishna, Wiita, P. J., 1991, ApJ, 373, 325

Gurvits, L. I., 1994, ApJ, 425, 442

Kapahi V. K., 1975, MNRAS, 172, 513

Kapahi, V. K., 1989, AJ, 97,1

Kapahi V. K., Athreya R. M., Subrahmanya C. R., Hunstead R. W., Baker J. C., McCarthy P. J., van Breugel W., 1994, JA\&A, in press

Kellermann, K. I., 1993, Nature, 361, 134

Komberg, B. V., Kravtsov, A. V., Lukash, V. N., 1994, A\&A, 286, L19

Laing, R. A., Riley, J. M., Longair, M. S., 1983, MNRAS, 204, 151

Legg, T. H., 1970, Nature, 226, 65

Miley, G. K., 1968, Nature, 218, 933

Miley, G. K., 1971, MNRAS, 152, 477

Nilsson, K., Valtonen M. J., Kotilainen J., Jaakkola T., 1993, ApJ, 413, 453

Oort, M. J. A., Katgert, P., Windhorst, R. A., 1987, Nature, 328, 500

Orr, M. J. L., Browne, I. W. A., 1982, MNRAS, 200, 1067

Pearson, T. J., Xu, W., Thakkar, D. D., Readhead, A. C. S., Polatidis, A. G., Wilkinson, P. N., 1994, in Compact Extragalactic radio sources, Proc. Socorro Workshop, eds. Zensus, J. A., Kellermann, K. I., NRAO, p. 1

Shaver, P. A., 1988, in Large Scale Structure of the Universe, ed. Audouze J., Pelletan, M.-C., Szalay, A., Kluwer, Dordrecht, p. 359

Shaver, P. A., 1992, Physica Scripta, T43, 51

Singal, A. K., 1988, MNRAS, 233, 87

Singal, A. K., 1993, MNRAS, 263, 139

Swarup, G., 1975, MNRAS, 172, 501 